\title{
Knee Rotation in Relation to Laterality, Sex, Applied Torque, and Knee Angle
}

\author{
Seiya Masegi ${ }^{1}$, Hitoshi Shiraki ${ }^{2}$ and Shumpei Miyakawa ${ }^{2}$ \\ 1. Department of Health and Sports, Faculty of Health Sciences, Niigata University of Health and Welfare, Niigata 950-3198, Japan \\ 2. Faculty of Health and Sport Sciences, University of Tsukuba, Tsukuba, Ibaraki 305-8577, Japan
}

\begin{abstract}
Rotational knee laxity is known as an intrinsic risk factor for anterior cruciate ligament injury. However, there was no convenient way to measure rotational range of motion. To this end, we developed the RotorMeter, a non-invasive external device intended to measure rotational range of motion of the knee. The purpose of this study is to use the RotorMeter to clarify the characteristics pertaining to the rotational range of motion of knee in healthy participants under varying conditions. A total of 23 healthy participants participated in the study. Torques of $2.5,5.0$, and $7.5 \mathrm{Nm}$ were applied at $90^{\circ}, 60^{\circ}$, and $30^{\circ}$ angles of knee flexion to measure the rotational range of motion of knee using the RoterMeter. Female participants showed larger rotational range of motion than the males, and no laterality was observed at any flexion angle and applied torque. When different torques were applied at the same flexion angle, rotational range of motion significantly increased at all flexion angles with increased torque. When the same torque was applied at different flexion angles, no significant difference in rotational range of motion was observed at any of the flexion angles.
\end{abstract}

Key words: Knee, rotation, RotorMeter.

\section{Introduction}

The knee is the joint that bears the largest load in the human body, and it plays a particularly important role in sports activities. Knee injuries due to sports activities are common [1]. In anterior cruciate ligament (ACL) injuries, the time, psychological, financial, and social losses suffered by the patients are enormous, even though advances in ligament reconstruction and postoperative athletic rehabilitation have made the return to highly competitive sports possible [2-5]. Thus, preventing ACL injuries is a matter of serious concern for people involved in athletic training.

Of the ACL injuries, noncontact injuries are known to be more common than contact injuries [6], and intrinsic risk factors for noncontact injuries include joint laxity, static and dynamic alignment of lower extremity, size of the ACL, and sex differences [7]. Regarding joint laxity, there have been reports focused on generalized joint laxity as well as laxity of the knee

Corresponding author: Seiya Masegi, MSc, associate professor, research field: athletic training. joint itself. ACL injuries have been reported to occur more commonly in people with valgus knee, hyperextended knee, and knees with increased anterior translation of the tibia relative to the femur [8,9]. However, although there have been reports of malalignment in cases of knee rotation at the time of the occurrence of ACL injuries [10-12], there have been no reports on the association between range of motion in a rotational direction and ACL injuries; perhaps because no method has been established for the measurement of the rotational range of motion of knee.

Previous studies on the rotational range of motion of knee have used cadaveric knees [13, 14]; however, soft tissue degeneration in cadaveric knees makes it difficult to compare them with the knees in vivo. In addition, although few research studies conducted on the knees in vivo have been reported [15-17], methods for the detection of the rotational range of motion of knee, limb posture during the measurements, and applied torque were not standardized, and the number of samples was relatively small; as a result, no consensus has yet been reached. Therefore, we 
developed a measuring instrument called the RotorMeter and reported its statistical reliability and validity as an instrument for measuring rotational range of motion of knee [18].

The purpose of this study was to measure the rotational range of motion of knee in healthy adult females and males under different conditions using the RotorMeter and to acquire basic information pertaining to the rotational range of motion of knee.

\section{Materials and Methods}

\subsection{Measuring Instrument}

Fig. 1 shows the RotorMeter that was used in this study. The RotorMeter is composed of 3 parts: the bed, the rotor, and the console.

The bed is made of a flat aluminum plate fixed to a square aluminum frame, to which is attached a thigh-board with an angle that can be adjusted by $15^{\circ}$ intervals (Fig. 2). Attached to the thigh-board are 2 clamps for femoral condyle fixation that can optionally fix the thigh-board in position, 2 Velcro tapes for femoral fixation, and 2 calf rests.

On the rotor, a plastic disk capable of rotating freely is attached to the aluminum board, and a plastic boot with built-in 4 airbags, used to hold the foot in place, is attached to the disk. The axis of the boot is fixed in the direction of the long axis of the lower extremities and is consistent with the rotational axis of the disk.

A rotary encoder designed to convert the amount of disk rotation into electric signal is attached to the disk's rotational axis. A steel wire is attached to the circumference of the disk and guided downward by a fixed pulley attached to the lateral side of the disk. A weight designed for applying torque is attached to the tip of the wire. In addition, the direction of the load can be set to both internal and external by changing the position of the junction between the wire and the disk. The position of the entire rotor can be moved upward, downward, or toward the right or left side by operating the handles placed at 2 locations.

A power supply and board designed to process the electrical signal from the rotary encoder are installed inside the console, and a touchscreen liquid crystal display showing various measurement data and a power switch are installed at the surface of the console. A cable is pulled out from the console, and a hand switch for notification of the beginning of the torque load is attached to the tip of the cable.

\subsection{Participants}

The study was conducted on 11 females and 12 males with no history of severe traumatic injury of the lower extremities or pelvic girdle, and the measurements were carried out on both the right and left legs. The participants' physical characteristics were as follows: in the female group, average age was $21.2 \pm 1.03$ years, average height $160.8 \pm 5.70 \mathrm{~cm}$, and average weight $53.6 \pm 5.09 \mathrm{~kg}$ and in the male group,

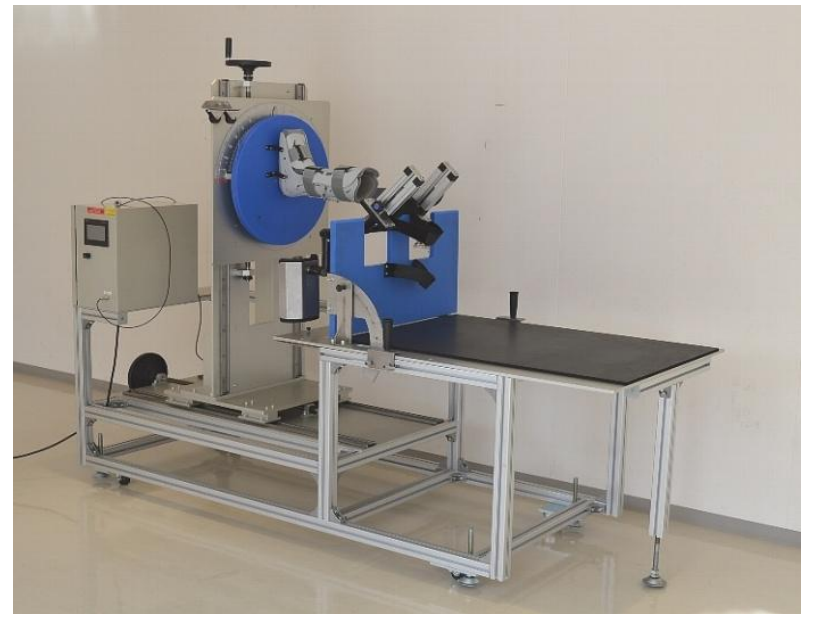

Fig. 1 The RotorMeter.

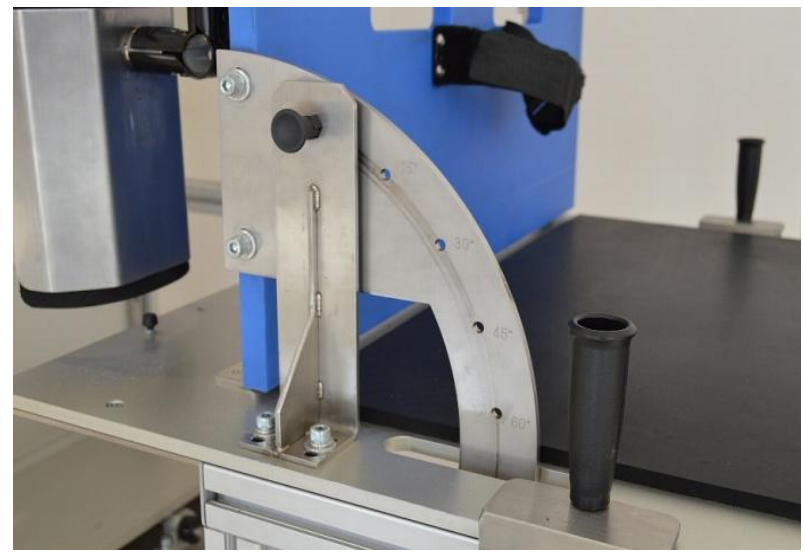

Fig. 2 The hinge of the thigh-board. 
average age was $21.3 \pm 1.09$ years, average height $173.3 \pm 6.56 \mathrm{~cm}$, and average weight $67.8 \pm 8.02 \mathrm{~kg}$.

\subsection{Measurement Method}

Fig. 3 shows the participant's posture during the measurements. The participant's thigh, on the measurement side, was fixed to the thigh-board using 2 Velcro tapes, and the medial and lateral femoral epicondyles were fixed in place with clamps (Fig. 4). On this occasion, findings confirmed that, in the horizontal plane, the participants' thigh of the measurement side was perpendicular to the bed. Then the foot and the lower leg on the measurement side were inserted into the boot and held in place by inflating the airbags with a hand pump (Fig. 5). On the non-measurement side, the participants were instructed to place their leg on the calf rest. Before applying torque,

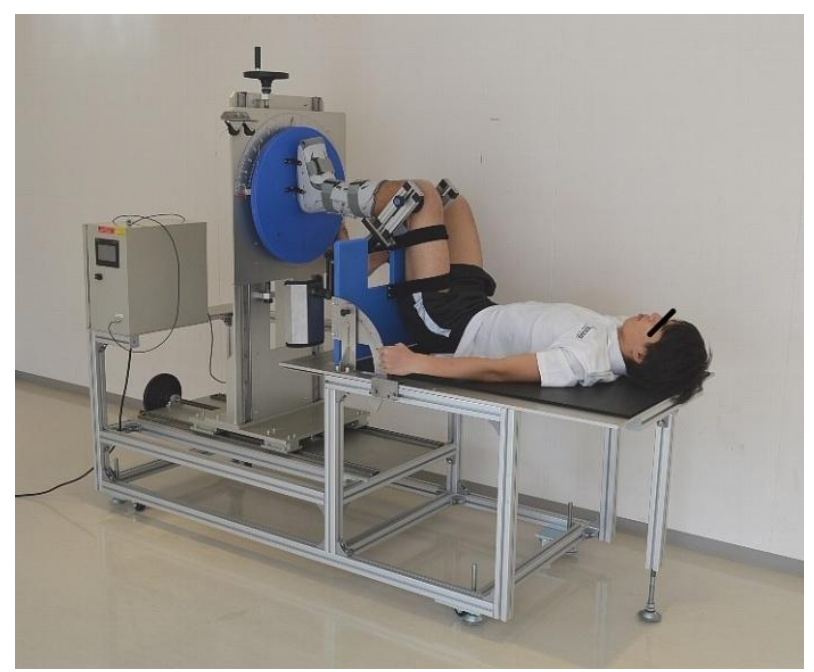

Fig. 3 The measurement position at $90^{\circ}$ of knee flexion.

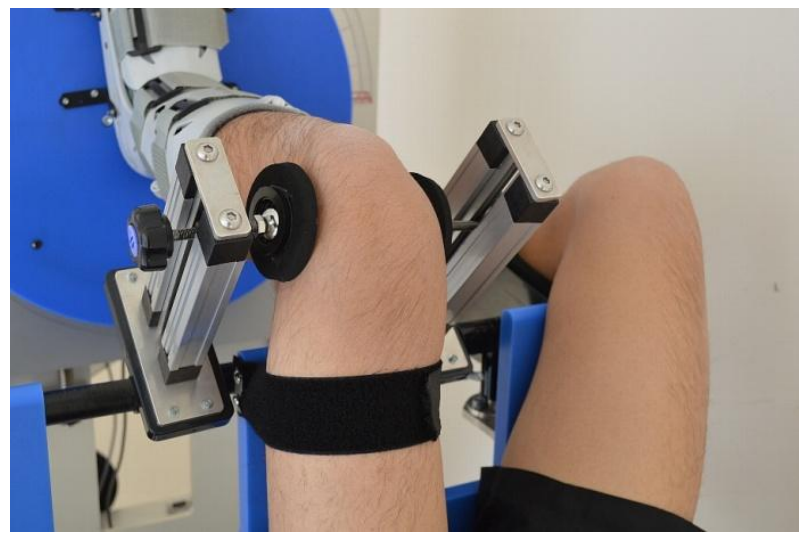

Fig. 4 Fixation of thigh and knee. the disk was fixed using the locking mechanism to ensure that the long axis of the foot was vertical, and this position was defined as the neutral position.

The knee flexion angles at the time of the measurement were set to $30^{\circ}, 60^{\circ}$, and $90^{\circ}$, and the applied torques were 2.5, 5.0 and $7.5 \mathrm{Nm}$, using weights. Conducted under a total of 9 conditions using a combination of 3 different angles with 3 different applied torques, the measurements were carried out randomly using internal and external rotation as directions of rotation (Figs. 6a and 6b). Total (internal + external) rotational range of motion was obtained.

During the measurements, the participants were instructed to relax the muscles in both lower extremities.

The system was programmed in such a way that the measured value shown on the display was fixed at the beginning of torque loading, in other words, 20 seconds

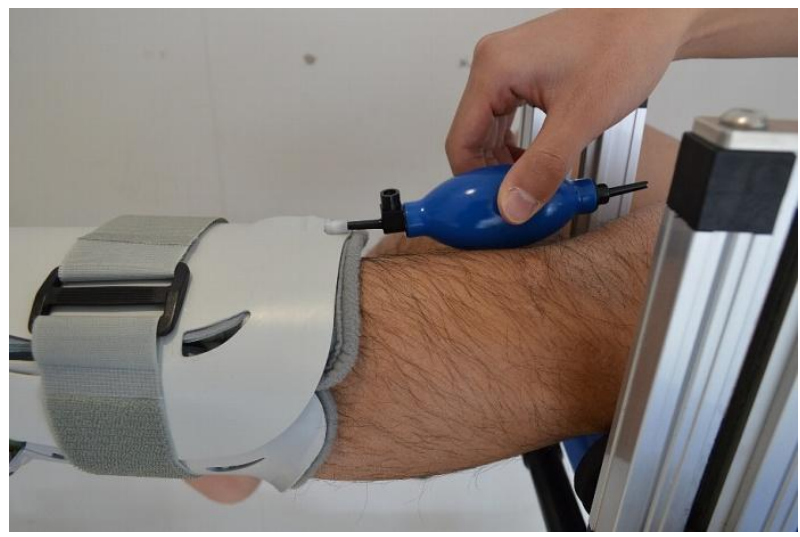

Fig. 5 Inflating air into airbag with hand-pump.

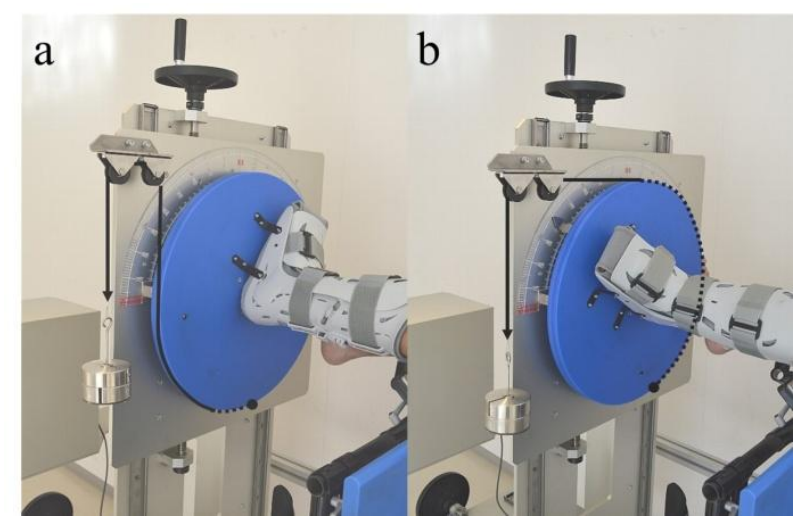

Fig. 6 The disk and the boot during internal rotation (a) and external rotation (b) of left knee. The wire is shown as bold/dot lines. 
Table 1 Total rotation with $2.5,5.0$ and $7.5 \mathrm{Nm}$ torques measured at $90^{\circ}, 60^{\circ}$ and $30^{\circ}$ of knee flexion on the right and left knees of female and male (mean $\pm \mathrm{SD})$.

\begin{tabular}{|c|c|c|c|c|c|c|c|c|c|}
\hline \multirow{3}{*}{\multicolumn{2}{|c|}{$\begin{array}{c}\text { Knee Flexion } \\
\text { Angle and } \\
\text { Applied Torque }\end{array}$}} & \multicolumn{2}{|c|}{ Total Rotation -Right $\left({ }^{\circ}\right)$} & \multicolumn{2}{|c|}{ Total Rotation -Left $\left({ }^{\circ}\right)$} & \multicolumn{4}{|c|}{$P$ - value } \\
\hline & & \multirow{2}{*}{ Female } & \multirow{2}{*}{ Male } & \multirow{2}{*}{ Female } & \multirow{2}{*}{ Male } & \multicolumn{2}{|c|}{ Sex } & \multicolumn{2}{|c|}{ Laterality } \\
\hline & & & & & & Right & Left & Female & Male \\
\hline \multirow{3}{*}{$90^{\circ}$} & $2.5 \mathrm{Nm}$ & $79.2 \pm 18.7$ & $55.3 \pm 8.2$ & $81.3 \pm 8.4$ & $56.8 \pm 9.6$ & 0.000 & 0.000 & 0.602 & 0.445 \\
\hline & $5.0 \mathrm{Nm}$ & $99.8 \pm 11.1$ & $76.1 \pm 9.3$ & $100.0 \pm 10.2$ & $79.6 \pm 10.8$ & 0.000 & 0.000 & 0.941 & 0.201 \\
\hline & $7.5 \mathrm{Nm}$ & $118.0 \pm 15.0$ & $92.3 \pm 9.5$ & $117.1 \pm 12.1$ & $94.5 \pm 11.5$ & 0.000 & 0.000 & 0.730 & 0.294 \\
\hline \multirow{3}{*}{$60^{\circ}$} & $2.5 \mathrm{Nm}$ & $73.0 \pm 11.7$ & $51.0 \pm 8.1$ & $76.0 \pm 16.2$ & $51.6 \pm 7.3$ & 0.000 & 0.000 & 0.329 & 0.830 \\
\hline & $5.0 \mathrm{Nm}$ & $93.2 \pm 9.0$ & $75.1 \pm 9.5$ & $96.5 \pm 15.5$ & $73.8 \pm 11.8$ & 0.000 & 0.001 & 0.270 & 0.595 \\
\hline & $7.5 \mathrm{Nm}$ & $112.0 \pm 11.4$ & $90.5 \pm 9.9$ & $113.1 \pm 13.7$ & $90.8 \pm 11.3$ & 0.000 & 0.000 & 0.518 & 0.896 \\
\hline \multirow{3}{*}{$30^{\circ}$} & $2.5 \mathrm{Nm}$ & $66.4 \pm 14.6$ & $51.0 \pm 9.2$ & $64.0 \pm 7.8$ & $47.8 \pm 8.8$ & 0.008 & 0.000 & 0.535 & 0.282 \\
\hline & $5.0 \mathrm{Nm}$ & $92.2 \pm 15.2$ & $73.0 \pm 11.4$ & $89.6 \pm 13.5$ & $77.5 \pm 11.1$ & 0.003 & 0.036 & 0.551 & 0.115 \\
\hline & $7.5 \mathrm{Nm}$ & $116.0 \pm 17.9$ & $91.6 \pm 13.1$ & $107.1 \pm 10.3$ & $89.1 \pm 11.8$ & 0.002 & 0.001 & 0.076 & 0.467 \\
\hline
\end{tabular}

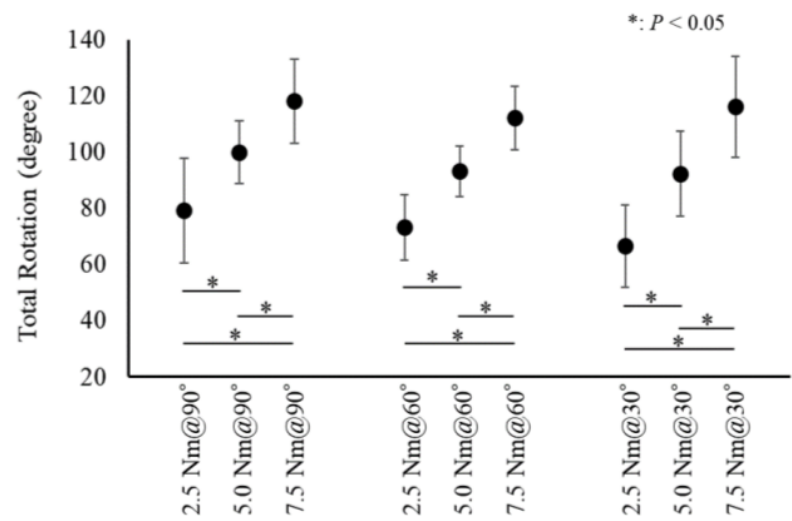

Appiled Torque and Knee Angle

Fig. 7 Total rotation with $2.5,5.0$ and $7.5 \mathrm{Nm}$ torques at $90^{\circ}, 60^{\circ}$ and $30^{\circ}$ of knee flexion angle on right knee of female.

after input of the signal from the hand switch.

All measurements were discontinued immediately whenever a participant complained of pain or discomfort.

\subsection{Statistical Analysis}

Laterality was examined using the paired $t$-test; sex difference was examined using Student's $t$-test; repeated measure ANOVA was used to compare joint angle conditions and applied torque conditions; and when an interaction was found, Bonferroni post-hoc analysis was used. Stoical analysis was performed using IBM SPSS Statistics v20.0, and the statistical significance level was set at $5 \%$.

\subsection{Ethical Considerations}

This study followed the Declaration of Helsinki and was prior approved by the ethics committee of the Niigata University of Health and Welfare (17503-14701). The participants received a verbal and written description of the study and provided informed consent.

\section{Results}

3.1 Laterality in the Rotational Range of Motion of Knee

Under all conditions, no significant laterality in the rotational range of motion of knee was found in either the male or female group (Table 1).

\subsection{Sex Difference in the Rotational Range of Motion of} Knee

Under all conditions, the rotational range of motion of knee was significantly greater in the female group than that in the male group (Table 1). 


\subsection{Rotational Range of Motion of Knee with Changes in the Applied Torque}

At all knee flexion angles, findings in both the male and female groups showed that the rotational range of motion of knee was significantly greater with a load of $5.0 \mathrm{Nm}$ than that with $2.5 \mathrm{Nm}$ and with a load of 7.5 $\mathrm{Nm}$ than that with $5.0 \mathrm{Nm}$ (Fig. 7).

\subsection{Rotational Range of Motion of Knee with Changes in Knee Flexion Angle}

Under all applied torque conditions, findings in both the male and female groups showed that changes in knee flexion angle caused no significant difference in the rotational range of motion of knee (Fig. 7).

\section{Discussion}

Quantitative determination and measurement of the range of motion and laxity of all joints in the human body are regarded as important, not only in the field of sports medicine, but also in other fields such as biomechanics and conditioning. Furthermore, elucidating their association with various manual tests used in clinical settings may provide objective proof supporting the results of those tests.

ACL injuries account for a large percentage of sport-related knee traumas [1]. Manual tests used for the diagnosis of ACL injuries include the Lachman test and the anterior drawer test for the evaluation of anteroposterior laxity of the tibia relative to the femur, and their quantitative validity has been demonstrated using instruments such as the KT-1000/2000 [19, 20]. In contrast, the pivot shift test and the dial test have been used as manual tests for evaluation of the tibia's rotational laxity relative to the femur [21, 22], but the means to perform a quantitative study of their validity is lacking, and evaluation of the results of the manual tests is believed to be dependent on the examiner's clinical experience [15]. Thus, there is a need to develop reliable measurement methods that allow for quantification and evaluation of the rotational range of motion of knee.
Previous studies have focused on the rotational range of motion of knee using a method similar to our current study. Among them, Shultz et al. [15] used the Vermont Knee Laxity Device and reported that the rotational range of motion of knee was $27.5^{\circ} \pm 7.5^{\circ}$ when a $5-\mathrm{Nm}$ torque was applied in supine position while the knee was in $20^{\circ}$ flexion. In a study using the Rotameter, Lorbach et al. [16] reported that the rotational range of motion of knee was $95.0^{\circ} \pm 3.5^{\circ}$ in prone position when the applied torque was $10 \mathrm{Nm}$ and the knee was in $30^{\circ}$ flexion. In a study using the Rottometer, Almquist et al. [17] reported that the rotational range of motion of knee was $78^{\circ} \pm 16^{\circ}$ when the applied torque of $9 \mathrm{Nm}$ was used and the knee was in $30^{\circ}$ flexion and that the rotational range of motion of knee was $77^{\circ} \pm 11^{\circ}$ when the same applied torque was used and the knee was in $90^{\circ}$ flexion. Those findings suggested that in the measurement of the rotational range of motion of knee, measurement results may be affected by the measuring instrument, the posture during the measurements, the applied torque, torque application method, and other measurement conditions.

In our study, which used the RotorMeter for making measurements, instructions regarding limb posture during the measurements were simple and weights were used for applying torque; as a result, no variation occurred during the measurements. In addition, the body weight loading condition was "non-load", and a torque application time of 20 seconds was prescribed. Furthermore, no special skill was needed to operate the RotorMeter and conduct the measurements implying that opportunities for measurement errors were kept to a minimum. Therefore, the RotorMeter is arguably a highly effective instrument for the measurement of the rotational range of motion of knee.

Our results showed no difference laterality in the rotational range of motion of knee in healthy adults regarding sex. This finding is similar to that reported by Nawata et al. [23] in their study on the amount of anterior displacement of the tibia. Our findings also 
showed that, under all conditions, the rotational range of motion of knee was significantly greater in females than in males. This was consistent with the findings of a study by Rozzi et al. [24], in which the amount of anterior displacement of the tibia was reportedly significantly greater in females than in males. These findings suggest that laterality and sex differences in the rotational range of motion of knee may be similar to those for the amount of anterior displacement of the tibia relative to the femur. In a prospective study, Uhorchak et al. [25] previously reported that the amount of anterior displacement of the tibia was greater in the ACL injury group than in the non-injury group. This suggests that the rotational range of motion of knee, with characteristics similar to those of the amount of anterior displacement of the tibia, could be useful as an indicator in the screening of a population at risk for ACL injury.

In our study, the results of measurements conducted using the same knee flexion angle while changing the applied torque were $2.5 \mathrm{Nm}<5.0 \mathrm{Nm}<7.5 \mathrm{Nm}$ both in females and males. Almquist et al. [26] previously reported that, when the knee flexion angle was changed to $30^{\circ}, 60^{\circ}$, and $90^{\circ}$, the rotational range of motion of knee was $6 \mathrm{Nm}<9 \mathrm{Nm}$ at all knee flexion angles. This was supported by the findings of our study. The findings of our pilot study showed that when the applied torque was set to $10 \mathrm{Nm}$, there were fewer cases of instability between the clamps at the distal end of the thigh. In addition, our study showed several occurrences of phenomena similar to those mentioned above when a load of $7.5 \mathrm{Nm}$ was applied. This suggested that, when using a RotorMeter, an applying torque of $5.0 \mathrm{Nm}$ might be appropriate for the measurement of the rotational range of motion of knee.

The ligaments of the knee joint are known to become tense at a given knee flexion angle. Sakane et al. [27] reported that for the ACL, the anteromedial fiber bundle reaches a maximum tension at $15^{\circ}$ flexion, the posterolateral fiber bundle reaches a maximum tension at $60^{\circ}$ to $90^{\circ}$ flexion, and the entire ACL reaches a maximum tension at $15^{\circ}$ flexion. In addition, Park et al. [28] reported that, under conditions using $90^{\circ}, 60^{\circ}, 30^{\circ}$, and $0^{\circ}$ knee flexion angle, maximum extension of the medial collateral ligament of knee was seen at a knee flexion angle of $0^{\circ}$. Based on the above, we predicted that the rotational range of motion of knee would be affected by the knee flexion angle, and the range of rotational motion of knee would decrease particularly when the knee was at a near complete extension. However, in our study, the rotational ranges of motion of knee at knee flexion angles of $90^{\circ}, 60^{\circ}$, and $30^{\circ}$ were compared using the same applied torque, but no significant difference was found. This finding supported those previously reported by Almquist et al. [26]. However, Lehmkuhl and Smith [29] and Levangie and Norkin [30] reported that the rotational range of motion of knee was affected by the knee flexion angle; showing that no consensus had been reached even in previous studies. The range of motion of knee is believed to be affected by not only the tension of each ligament but also the morphology of the articular surfaces of the femur and tibia on opposite sides, as well as the extensibility of the joint capsule, muscles, and tendons, and the mobility of the menisci on the tibial plateau. For this reason, the association between the knee flexion angle and the rotational range of motion of knee will need to be further investigated in the future.

During the measurements in our study, the foot and lower leg were fixed to the disk using plastic boot; the thigh was fixed to the thigh-board using Velcro tapes; and the medial and lateral femoral epicondyles were also fixed to the thigh-board using clamps. But the foot and the lower leg that was inserted inside the boot were fixed in place only using pressurization through injection of air into the boot's built-in airbags; therefore, the movements of various joints around the ankle may have not been sufficiently restricted. This may explain why adduction and abduction of the subtalar joint and talocrural joint were induced when torque was applied. In addition, this can also be 
presumed to have induced mobility of the intertarsal joints, tarsal bones, and intermetatarsal joints, as well as deformation of the soft tissue. This suggests that the values obtained in this study were a combination of the rotational range of motion of knee plus those of the ankle joint and other joints of the foot. In addition, it can also be presumed that the measurement results may have been affected by differences between participants in terms of soft tissue volume and extensibility. However, this might be unavoidable in noninvasive measurement tools, like the RotorMeter used in this study, which do not use bone pins. Measurement methods using multiple clamps for strong fixation of the lower leg, ankle, and foot are also conceivable, but due to pain and discomfort, the lower-limb muscle groups would become tense, which would also affect the measurement results. Considering these points, we suggest that in the use of the RotorMeter for the measurement of the range of knee joint rotation, there is room for discussion on improving the methods of fixation of the foot, ankle joint, and lower leg.

\section{Study Limitations}

In this study, the number of participants (11 females and 12 males) was relatively small, and the participants' age-range was limited. Therefore, for further validation, measurements may need to be carried out with a larger number of participants.

In female participants, the menstrual cycle at the time of measurement was not standardized. Deie et al. [31] and Heitz et al. [32] reported that the amount of anterior translation of the tibia was different depending on the menstrual cycle. On the other hand, Karageanes et al. [33] reported that no significant difference was found between the amount of anterior displacement of the tibia at the 3 phases of the menstrual cycle, namely, the follicular phase, the ovulation phase, and the luteal phase. Although the association between the rotational range of motion of knee and menstrual cycle remains unclear, conducting measurements using a standardized menstrual cycle would be valuable.
Further, because the measurements in this study were carried out with participants in the supine position, the condition pertaining to the body weight load on the knee joint was "non-load". However, most sport-related injuries affecting the knee joints, such as ACL injuries, are believed to occur at the location of the load; therefore, measurements at the location of the load using a modified RotorMeter may also be important.

\section{Conclusions}

The RotorMeter was used to clarify the characteristics pertaining to the rotational range of motion of knee in healthy participants under varying conditions. The female participants showed larger rotational range of motion than the males, and no laterality was observed at any flexion angle and applied torque. When different torques were applied at the same flexion angle, the rotational range of motion significantly increased at all flexion angles with increased torque. When the same torque was applied at different flexion angles, no significant difference in rotational range of motion was observed at any of the flexion angles.

\section{Acknowledgments}

This study was carried out upon receiving subsidy from the Niigata University of Health and Welfare within the research subsidy program (grant-in-aid for exploratory research) of 2013. The authors have no conflicts of interests to disclose regarding this study.

\section{References}

[1] Loes, M. D., Dahlstedt, L. J., and Thomee, R. 2000. “A 7-Year Study on Risks and Costs of Knee Injuries in Male and Female Youth Participants in 12 Sports." Scandinavian Journal of Medicine \& Science in Sports 10 (2): 90-7.

[2] Hewett, T. E., Lindenfeld, T. N., Riccobene, J. V., and Noyes, F. R. 1999. "The Effect of Neuromuscular Training on the Incidence of Knee Injury in Female Athletes." The American Journal of Sports Medicine 27 (6): 699-706. 
[3] Freedman, K. B., Glasgow, M. T., Glasgow, S. G., and Bernstein, J. 1998. "Anterior Cruciate Ligament Injury and Reconstruction among University Students." Clinical Orthopaedics and Related Research 356: 208-12.

[4] Smith, A. M., Scott, S. G., and Wiese, D. M. 1990. "The Psychological Effects of Sports Injuries Coping." Sports Medicine 9 (6): 352-69.

[5] Arendt, E., and Dick, R. 1995. "Knee Injury Patterns among Men and Women in Collegiate Basketball and Soccer: NCAA Data and Review of Literature." The American Journal of Sports Medicine 23 (6): 694-701.

[6] Agel, J., Arendt, E. A., and Bershadsky, B. 2005. "Anterior Cruciate Ligament Injury in National Collegiate Athletic Association Basketball and Soccer." The American Journal of Sports Medicine 33 (4): 524-31.

[7] Ireland, M. L. 1999. “Anterior Cruciate Ligament Injury in Female Athletes: Epidemiology." Journal of Athletic Training 34 (2): 150.

[8] Loudon, J. K., Jenkins, W., and Loudon, K. L. 1996. "The Relationship between Static Posture and ACL Injury in Female Athletes." Journal of Orthopaedic \& Sports Physical Therapy 24 (2): 91-7.

[9] Ramesh, R., Von Arx, O., Azzopardi, T., and Schranz, P. J. 2005. "The Risk of Anterior Cruciate Ligament Rupture with Generalised Joint Laxity.” Bone \& Joint Journal 87 (6): 800-3.

[10] Arnold, J. A., Coker, T. P., Heaton, L. M., Park, J. P., and Harris, W. D. 1979. "Natural History of Anterior Cruciate Tears." The American Journal of Sports Medicine 7 (6): 305-13.

[11] McNair, P. J., Marshall, R. N., and Matheson, J. A. 1990. "Important Features Associated with Acute Anterior Cruciate Ligament Injury." The New Zealand Medical Journal 103 (901): 537-9.

[12] Olsen, O. E., Myklebust, G., Engebretsen, L., and Bahr, R. 2004. "Injury Mechanisms for Anterior Cruciate Ligament Injuries in Team Handball." The American Journal of Sports Medicine 32 (4): 1002-12.

[13] Lane, J. G., Irby, S. E., Kaufman, K., Rangger, C., and Daniel, D. M. 1994. "The Anterior Cruciate Ligament in Controlling Axial Rotation: An Evaluation of Its Effect." The American Journal of Sports Medicine 22 (2): 289-93.

[14] Magit, D. P., McGarry, M., Tibone, J. E., and Lee, T. Q. 2008. "Comparison of Cutaneous and Transosseous Electromagnetic Position Sensors in the Assessment of Tibial Rotation in a Cadaveric Model." The American Journal of Sports Medicine 36 (5): 971-7.

[15] Shultz, S. J., Shimokochi, Y., Nguyen, A. D., Schmitz, R. J., Beynnon, B. D., and Perrin, D. H. 2007. "Measurement of Varus-Valgus and Internal-External Rotational Knee Laxities in vivo-Part I: Assessment of Measurement
Reliability and Bilateral Asymmetry." Journal of Orthopaedic Research 25 (8): 981-8.

[16] Lorbach, O., Wilmes, P., Theisen, D., Brockmeyer, M., Maas, S., Kohn, D., and Seil, R. 2009. "Reliability Testing of a New Device to Measure Tibial Rotation." Knee Surgery, Sports Traumatology, Arthroscopy 17 (8): 920-6.

[17] Almquist, P. O., Arnbjörnsson, A., Zätterström, R., Ryd, L., Ekdahl, C., and Fridén, T. 2002. "Evaluation of an External Device Measuring Knee Joint Rotation: An in vivo Study with Simultaneous Roentgen Stereometric Analysis." Journal of Orthopaedic Research 20 (3): 427-32.

[18] Masegi, S., Kanamori, A., Shiraki, H., and Miyakawa, S. 2016. "Evaluation of Reliability and Validity of a New Device to Measure Knee Rotation." Japanese Journal of Clinical Sports Medicine 24 (2): 261-8. (in Japanese)

[19] Daniel, D. M., Malcom, L. L., Losse, G., Stone, M. L., Sachs, R., and Burks, R. 1985. "Instrumented Measurement of Anterior Laxity of the Knee." JBJS 67 (5): 720-6.

[20] Highgenboten, C. L., Jackson, A., and Meske, N. B. 1989. "Genucom, KT-1000, and Stryker Knee Laxity Measuring Device Comparisons: Device Reproducibility and Interdevice Comparison in Asymptomatic Subjects." The American Journal of Sports Medicine 17 (6): 743-6.

[21] Bach Jr, B. R., Warren, R. F., and Wickiewicz, T. L. 1988. "The Pivot Shift Phenomenon: Results and Description of a Modified Clinical Test for Anterior Cruciate Ligament Insufficiency." The American Journal of Sports Medicine 16 (6): 571-6.

[22] Noyes, F. R., Grood, E. S., Cummings, J. F., and Wroble, R. R. 1991. "An Analysis of the Pivot Shift Phenomenon: The Knee Motions and Subluxations Induced by Different Examiners." The American Journal of Sports Medicine 19 (2): 148-55.

[23] Nawata, K., Teshima, R., Morio, Y., Hagino, H., Enokida, M., and Yamamoto, K. 1999. "Anterior-Posterior Knee Laxity Increased by Exercise: Quantitative Evaluation of Physiologic Changes." Acta orthopaedica Scandinavica 70 (3): 261-4.

[24] Rozzi, S. L., Lephart, S. M., Gear, W. S., and Fu, F. H. 1999. "Knee Joint Laxity and Neuromuscular Characteristics of Male and Female Soccer and Basketball Players." The American Journal of Sports Medicine 27 (3): 312-9.

[25] Uhorchak, J. M., Scoville, C. R., Williams, G. N., Arciero, R. A., Pierre, P. S., and Taylor, D. C. 2003. "Risk Factors Associated with Noncontact Injury of the Anterior Cruciate Ligament." The American Journal of Sports Medicine 31 (6): 831-42.

[26] Almquist, P. O., Ekdahl, C., Isberg, P. E., and Fridén, T. 2013. "Knee Rotation in Healthy Individuals Related to 
Age and Gender." Journal of Orthopaedic Research 31 (1): 23-8.

[27] Sakane, M., Fox, R. J., Glen, S. L. Y. W., Livesay, A., Li, G., and Fu, F. H. 1997. "In situ Forces in the Anterior Cruciate Ligament and Its Bundles in Response to Anterior Tibial Loads." Journal of Orthopaedic Research 15 (2): 285-93.

[28] Park, S. E., DeFrate, L. E., Suggs, J. F., Gill, T. J., Rubash, H. E., and Li, G. 2006. "Erratum to 'the Change in Length of the Medial and Lateral Collateral Ligaments during in vivo Knee Flexion'.” The Knee 13 (1): 77-82.

[29] Lehmkuhl, L. D., and Smith, L. K. 1983. "Knee Region." In Brunnstrom's Clinical Kinesiology (4th ed.). Philadelphia: Davis Company, 287-307.

[30] Levangie, P. K., and Norkin, C. C. 2011. "The Knee Complex." In Joint Structure and Function: A
Comprehensive Analysis. Philadelphia: Davis Company, 337-2.

[31] Deie, M., Sakamaki, Y., Sumen, Y., Urabe, Y., and Ikuta, Y. 2002. "Anterior Knee Laxity in Young Women Varies with Their Menstrual Cycle." International Orthopaedics 26 (3): 154-6.

[32] Heitz, N. A., Eisenman, P. A., Beck, C. L., and Walker, J. A. 1999. "Hormonal Changes throughout the Menstrual Cycle and Increased Anterior Cruciate Ligament Laxity in Females." Journal of Athletic Training 34 (2): 144.

[33] Karageanes, S. J., Blackburn, K., and Vangelos, Z. A. 2000. "The Association of the Menstrual Cycle with the Laxity of the Anterior Cruciate Ligament in Adolescent Female Athletes." Clinical Journal of Sport Medicine 10 (3): $162-8$. 